\section{Second Conference of the British Occupational Hygiene Society}

\section{R. J. SHERWOOD}

The second full-day conference of the British Occupational Hygiene Society held on April 6, 1954, at the London School of Hygiene and Tropical Medicine was opened by the incoming President, Professor E. J. King, who paid a tribute to Dr. Bedford, the retiring President, for his work in founding the Society and ensuring the success of the first year of its life.

He expressed a welcome to all visitors, especially those from overseas.

The subject discussed at the conference was air contamination in industry and its effect on the health of workers. The first paper to be presented, "The Investigation of Atmospheric Contaminants in Factories ", was delivered in two parts.

In introducing the first section on fundamental aspects, Dr. M. W. Goldblatt, Head of the Industrial Hygiene Laboratories, I.C.I. Ltd., stressed that present standards of the medical supervision of workers must be considered minimal. While big organizations had safety and medical departments, this was not true of the 230,000 factories which employed fewer than 100 workers. The only medical contacts that the managements and workers of these factories had were with the appointed factory doctors, who dealt primarily with young persons and particular trades.

It was true that very few cases of poisoning were notified, but the statistics gave a wholly misleading picture of conditions since mild chronic poisoning was probably never reported. Modern medical diagnosis and treatment made it possible for men to stay at work even when absorbing toxic substances. It was probable that chronic poisoning was widely incident in industry.

To ensure that conditions were safe when an atmospheric contaminant might be present, it was essential that, wherever possible, quantitative measurements of the contaminant be made. Merely to show its presence or absence meant nothing. Where toxic materials were present in measurable amounts and were being absorbed by the work people not only had air samples to be taken but also adequate records had to be kept so that over a period of time it might be possible to correlate the clinical histories and environmental concentrations. There was no such thing as an "allowable" concentration ; or rather, the " allowable" concentration always had to be zero. In practice, it was necessary to accept a "design" concentration as a criterion for the engineer to use in the design of equipment. The target always ought to be zero.

Dr. Goldblatt warned his audience against the wrong use of tables of maximum allowable concentrations. In particular, it was misleading to compare materials having different effects. This had been done by listing substances in zones of equi-toxicity; that was by placing several materials having similar maximum allowable concentrations in each zone. Each zone might then contain materials as different as irritants and narcotics which, on the surface, appeared to be equally toxic. Nevertheless, the narcotic, possibly causing insidious, chronic poisoning might well be the greater menace to health. It was essential to take notice of the nature of the material in assessing the possible risk of exposure. It was also most dangerous to predict the toxicity of a material by chemical analogy with others having a similar structure.

The second section, " Practical Aspects ", was presented by Dr. J. C. Gage, Head of the Biochemical Section, Industrial Hygiene Laboratories, I.C.I. Ltd., who said that it was essential that analytical methods were applied wherever possible and in the correct manner to determine whether work people were absorbing toxic materials.

The main requirements for a method for general application in a factory were that it should be inexpensive, self-contained, and unobtrusive; it should give an immediate result, should be so simple and reliable that a laboratory assistant or foreman could operate it, and it should be adequately accurate and specific. Such tests were particularly useful for testing air purity in a confined space or tank before workmen went in.

Tests of this type were exemplified by the simple test paper or bubbler methods described in the leaflets, "Methods for the Detection of Toxic Gases in Industry". Recent years had seen the development of indicator tubes for carbon monoxide and other substances. It was hoped that this principle would have a wide application.

More complicated instrumental methods were available which included the mercury vapour detector using ultraviolet light absorption, and the infra-red analyser which was available for numerous materials. Some of these instruments were capable of giving a continuous written record of atmospheric concentrations over long periods of time, or could be designed to give an automatic warning if safe limits were exceeded. These instruments had a useful, though limited, application to atmospheric studies in factories.

The first afternoon paper, "The Measurement of Dust Exposure for the Control of Pneumoconiosis ", was presented by Dr. B. M. Wright, of the Pneumoconiosis Research Unit, who said that the present lack of agreed safe limits for dust exposure was due partly to lack of agreement on the methods of making the measurements which were required to establish such limits, and partly to the fact that measurements had not been made on a big enough scale.

To set up safe limits, the relationship of a certain level of exposure (affected by the concentration and composition of the respirable fraction of the airborne dust to which workers were exposed and the duration of their exposure), and the incidence and severity of pneumoconiosis amongst those exposed had to be determined. The level of exposure which would be accepted as safe would then depend on what incidence and severity of pneumoconiosis was considered tolerable.

It was essential that in measuring concentrations of dusts producing pneumoconiosis, only respirable dust be 
taken into account. Particles or aggregates above 5 or below 1 micron should not be included. The only instrument in common use that enabled this to be done was the thermal precipitator which collected the dust without breaking up the large particles or aggregates. Its chief handicap was that relatively short-term samples were taken and assessment of average conditions over a period of a week or more became laborious.

The essential requirements of a suitable sampling instrument were to enable an estimate to be made of the concentration and composition of airborne dust particles and aggregates in the respirable size range; to yield a sample which can be reasonably easily and accurately assessed ; to be capable of integrating its exposure over at least one working week ; to be cheap, robust, light, portable, self-contained, and automatic. Until such an instrument had been produced and used over a period of many years, safe limits of dust exposure could not be established with any precision.

Dr. Wright displayed several new pieces of sampling apparatus, including a double aspirator for the thermal precipitator, an automatic sedimentation cell, and a selective mass-sampler.

The final paper of the conference was presented by Dr. W. G. Marley, Head of the Division of Health Physics, Atomic Energy Research Establishment, Harwell, on " Permissible Levels of Exposure to Ionising Radiations and Radioactive Materials".

With the development of atomic energy and the use of artificial radioactive isotopes it had become necessary to examine early information on permissible levels of exposure to $x$ rays and gamma rays, and to extend their application to the new substances.

The work in various countries had been reviewed by the International Commission on Radiological Protection. At the 1953 meeting of the International Congress of Radiology, the Commission recommended values for the maximum permissible body burden of a wide range of isotopes and of the corresponding levels in air and drinking water. These were to be published shortly.

Dr. Marley discussed the methods of arriving at these recommended values. Wide experience with $x$ rays and gamma rays had made it possible to define a basic tolerance for external irradiation and this had been set at 0.3 roentgen/week in the tissue (corresponding in certain circumstances to approximately 0.5 roentgen/week measured with back-scatter on the surface of the body). A further basic tolerance figure from human exposure had been derived from the study of the effect of radium ingestion by radium dial painters, namely $0 \cdot 1$ microgram of radium fixed in the skeleton, which was thought to be safe over a working life time.

Different types of radiation or different isotopes could be compared experimentally and theoretically with these basic criteria, and the permissible level of exposure for the particular radiation or material could be calculated by allowing for the relative biological effectiveness of the particular radiation. Parameters that effected this calculation included the fractional uptake from lungs or gut, the metabolism of the element, the organ of concentration, the biological half-life, and the overall relative toxicity. These had been determined from animal studies and from a limited experience of accidental exposure in men.

To achieve agreement between the various laboratories undertaking research in this field, it was found necessary to define a "standard man" for whom the weight and constitution of various organs had been set. The levels recommended by the International Commission were intended for application to individuals who were exposed in their occupation. Should more than a small fraction of the population be exposed, the genetic effects would have to be considered.

Dr. Marley also discussed problems resulting from exposure due to accident or emergency, where a much more intense level had been present for a short period.

The conference was attended by 150 members of the Society and visitors. Copies of the proceedings will be available from the Hon. Secretary, Mr. Peter C. G. Isaac, Public Health Engineering Laboratory, King's College, Newcastle-upon-Tyne.

The next conference of the Society is to be held on November 1, 1954, at the London School of Hygiene and Tropical Medicine. This will be devoted to discussion of the hazards associated with radioactivity and the use of $x$ rays in industry.

Correction.-We regret that in the paper "Fatal Emphysema in Two Men Making a Copper Cadmium Alloy" by Ronald E. Lane and A. C. P. Campbell in the April issue an error was printed. On page 121 the cadmium concentrations should be stated in $\mu \mathrm{g}$. per $100 \mathrm{~g}$. fresh tissue and not $\mathrm{mg}$. per $100 \mathrm{~g}$. 